\section{Chlorophyll d as a major pigment}

SIR - Chlorophyll $d$ was reported first as a minor, green and magnesiumcontaining pigment in various species of red macroalgae in 1943 (ref. 1). It was thought by some, however, that chlorophyll $d$ could be an artefact produced by the pigment extraction process, as it is not found in all algae, and one of the oxidation derivatives of chlorophyll $a$ has an absorption spectrum identical with that of chlorophyll $\cdot d^{2,3}$. It remains unclear as to whether chlorophyll $d$ does occur in vivo. We have now isolated a previously undescribed oxygenic photosynthetic prokaryote containing chlorophyll $d$ as a major green pigment: it has only a small amount of chlorophyll $a$.

We isolated the new organism from a suspension of algae squeezed out of Lissoclinum patella, a colonial ascidian, collected in 1993 from the marine coast of the Palau islands in the western Pacific Ocean. Cells are unicellular and spheroidal or ellipsoidal, $1.5-2.0 \mu \mathrm{m}$ in diameter and $2.0-3.0 \mu \mathrm{m}$ in length. They are photoautotrophs, and have evolved in the presence of oxygen. We used electron

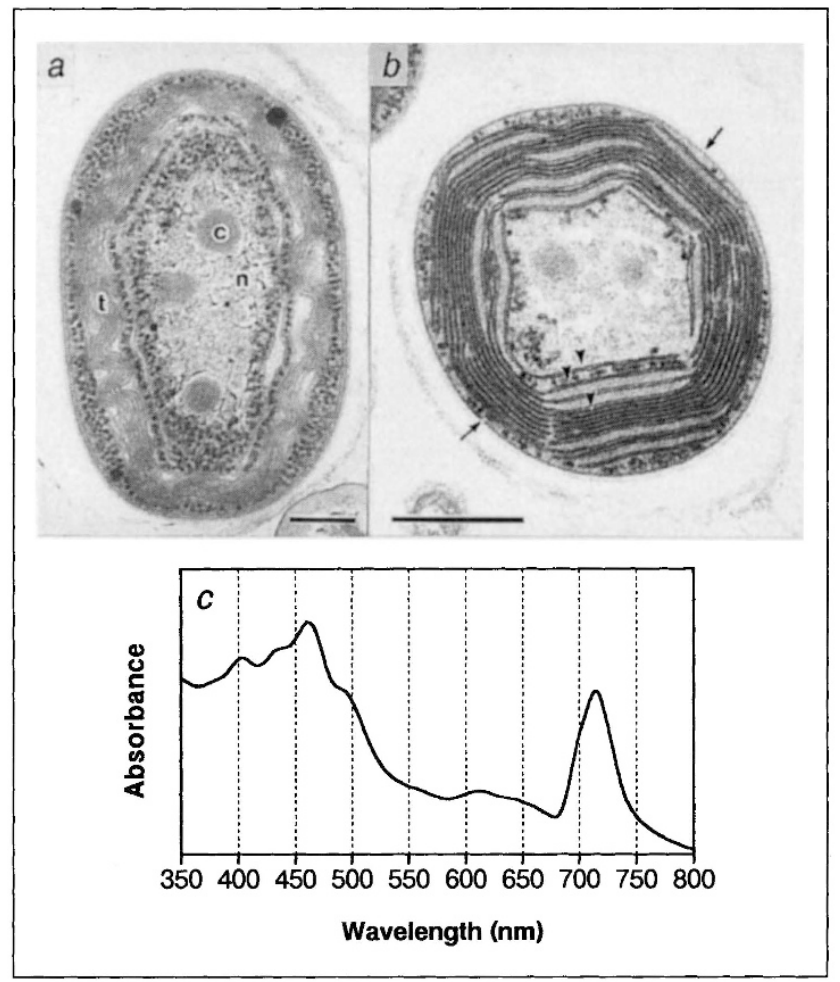

FIG. 1 a, Electron micrograph of a longitudinal section of a cell, showing the cytoplasmic space. The preparation was prefixed with $1 \%$ glutaraldehyde and postfixed with $2 \%$ osmic acid. c, carboxysome-like structure; $n$, nucleoide; $t$, thylakoid-like membranes. Scale bar, $0.3 \mu \mathrm{m}$. $b$, Electron micrograph of transverse section showing thylakoid-like membranes and plasmalemma. Cell prefixed with $1 \%$ glutaraldehyde and postfixed with $1 \%$ potassium permanganate: Arrowheads, thylakoid-like membranes; arrows, plasmalemma. Scale bar, $0.5 \mu \mathrm{m}$. $c$, Absorption spectrum of cells suspended in fresh culture medium. microscopy to demonstrate that the cell is a prokaryote (Fig. $1 a, b$ ). About 10 to membranes are stacked pherally (Fig. 1b), and wimilar to phycoon the membranes (Fig. 1a). These ultrastructural features are Prochlorophyta (Oxyacteria), oxygenic photosynthetic prokaryotes, containing chlorophylls $a$ biliproteins, rather than containphycobiliproteins.

The cell suspensions are green and have an absorption maximum in the red region at $714-718 \mathrm{~nm}$ (Fig. 1c), whereas all known oxygenic photosynthetic organmaximum around $675 \mathrm{~nm}$ due to a bathochromic shift of chlorophyll $a^{4}$. Pigment analysis by HPLC reveals that a green pigment with three absorption maxima at 400, 455 and $697 \mathrm{~nm}$ in methanol is a predominant pigment of the cell in addition to only a small amount of chlorophyll $a$. The absorption spectra of the predominant pigment and of its acidification product (peaks at 392,421 and $692 \mathrm{~nm}$ in diethyl ether) are the same as those reported for chlorophyll $d$ and phaeophytin $d^{1}$, respectively. We confirmed that the predominant pigment is chlorophyll $d$ by NMR and fast atom bombardment mass spectrometry (Fig. 2).

The chlorophyll $d$ makes up about $80 \%$ of the total lipidsoluble pigment of the cell and more than $2 \%$ of the cell dry weight. The amount of chlorophyll $a$, on the other hand, is less than $0.08 \%$ of the cell dry weight, whereas it ranges from $0.3 \%$ to $3 \%$ in other oxygenic photosynthetic organisms 5 . The ratio of chlorophyll $a$ to chlorophyll $d$ in the

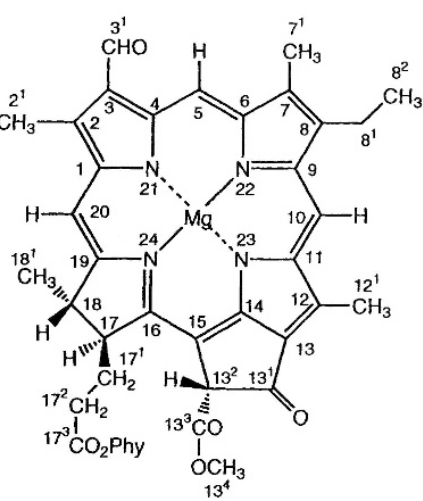

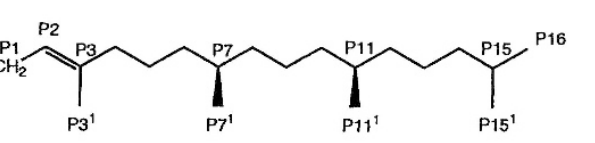

FIG. 2 Structure of chlorophyll $d$.

cells varies between 0.027 and 0.092 , depending on the culture conditions.

Our results confirm the natural occurrence of chlorophyll $d$ in a newly isolated, oxygenic, photosynthetic prokaryote. Moreover, the higher content of chlorophyll $d$ and the low ratio of chlorophyll $a$ to chlorophyll $d$ in this organism suggest the existence of a unique and as yet unknown light-harvesting system which uses chlorophyll $d$. We propose for the new organism the name Acaryochloris marina Miyashita et Chihara gen. et sp. nov., to be formally described elsewhere (manuscript in preparation).

Hideaki Miyashita

Hisato Ikemoto

Norihide Kurano

Marine Biotechnology Institute,

Kamaishi Laboratories,

Kamaishi,

Iwate 026, Japan

Kyoko Adachi

Marine Biotechnology Institute,

Shimizu Laboratories,

Shimizu,

Shizuoka 424-19, Japan

Mitsuo Chihara

Japanese Red Cross College of Nursing,

Shibuya-ku,

Tokyo 150, Japan

Marine Biotechnology Institute,

Kamaishi Laboratories

Kamaishi,

Iwate 026, Japan

Shigetoh Miyachi

Marine Biotechnology Institute,

Hongo, Bunkyo-ku,

Tokyo 113, Japan

e-mail: miya@mb.sendai.infoweb.or.jp

1. Manning, W. M. \& Strain, H. H. J. Biol. Chem. 151, 1-19 (1943).

2. Holt, A. S. \& Morley H. V. Can. J. Chem. 37, 507-514 (1959).

3. Holt, A. S. Can. J. Bot. 39, 327-331 (1961).

4. French, C. S., Brown, J. S. \& Lawrence, M. C. Plant Physiol. 49, 421-429 (1972).

5. Lee, R. E. in Phycology 2nd edn Ch. 1 (Cambridge Univ. Press, New York, 1989). 